\title{
Solubility of Anthracene in Binary and Ternary Mixtures of Cyclohexanone, Ethyl Acetate, and Methanol at 298.2 K
}

\author{
Abolghasem Jouyban, ${ }^{\dagger} *$ Jamshid L. Manzoori, ${ }^{\ddagger}$ Vahid Panahi-Azar, ${ }^{\S}$ Jafar Soleymani, ${ }^{\S}$ \\ Mohammad A. A. Fakhree," Ali Shayanfar, ${ }^{\perp}$ and William E. Acree, Jr."
}

Department of Pharmaceutical and Food Control, Faculty of Pharmacy, Drug Applied Research Center, Biotechnology Research Center, Tabriz University of Medical Sciences, Tabriz 51664, Iran, Department of Analytical Chemistry, Faculty of Chemistry, University of Tabriz, Tabriz, Iran, Kimia Research Institute, Tabriz, Iran, and Department of Chemistry, University of North Texas, Denton, Texas 76203-5070

The solubilities of anthracene in binary and ternary mixtures of cyclohexanone + ethyl acetate, cyclohexanone + methanol, ethyl acetate + methanol, and cyclohexanone + ethyl acetate + methanol were reported at $298.2 \mathrm{~K}$. The generated data were fitted to the Jouyban-Acree model, and the overall mean deviation was $5.1 \%$.

\section{Introduction}

Solubility data of polycyclic aromatic hydrocarbons (PAHs) have broad applications and great importance in the petroleum industry, such as the solvent selection for the reaction and separation processes. Also, some of the PAHs are known as carcinogenic agents ${ }^{1}$ thus, the data could be used to estimate environmental distribution and degradation of these compounds to follow up on these harmful chemicals. The solubility behavior of PAHs in a variety of solvent mixtures (e.g., binary and ternary mixtures) is very important because cosolvency is a common method to enhance the solubility and cosolvent blends are frequently used in purification methods. There is a significant lack of solubility data for most of the solutes; thus, efforts are devoted to represent a number of mathematical models for the solubility prediction of chemicals in multiple solvent mixtures, and a summary of the models was given in a recent paper. ${ }^{2}$

Anthracene, a simple PAH as a model solute, is the subject of a number of solubility studies, and experimental solubility data of anthracene in binary and ternary solvent mixtures have been reported in alcohols, ethers, alkenes, esters, and aromatic compounds by Acree and co-workers. ${ }^{3-9}$ The authors did not study the solubility of anthracene in solvent mixtures containing ketones, nor has the effect of interactions between ketone and alcohol and ester cosolvents been thoroughly investigated in regards to the solubility of a dissolved solute.

For solubility correlation or prediction in the solvent mixtures, one of the most accurate studied models is the Jouyban-Acree model. The Jouyban-Acree model is shown as ${ }^{10}$

\footnotetext{
* Author to whom correspondence should be addressed. E-mail: ajouyban@ hotmail.com. Fax: +98 4113363231.

Department of Pharmaceutical and Food Control, Faculty of Pharmacy.

$\doteqdot$ University of Tabriz.

$\S$ Drug Applied Research Center.

"Kimia Research Institute.

${ }^{\perp}$ Biotechnology Research Center.

\# University of North Texas.
}

$$
\ln C_{\mathrm{m}}^{\mathrm{Sat}}=x_{1} \ln C_{1}^{\mathrm{Sat}}+x_{2} \ln C_{2}^{\mathrm{Sat}}+x_{1} x_{2} \sum_{i=0}^{2} J_{i}\left(x_{1}-x_{2}\right)^{i}
$$

where $C_{\mathrm{m}}^{\text {Sat }}$ is the solute mole per liter solubility in the binary solvent mixtures; $x_{1}$ and $x_{2}$ are the mole fractions of solvents 1 and 2 in the absence of the solute; $C_{1}^{\text {Sat }}$ and $C_{2}^{\text {Sat }}$ denote the mole per liter solubility of the solute in neat solvents 1 and 2; and $J_{i}$ coefficients are the solvent-solvent and solute-solvent interaction terms. These constant terms could be calculated using a no-intercept least-squares regression of $\left(\ln C_{\mathrm{m}}^{\text {Sat }}-x_{1} \ln C_{1}^{\text {Sat }}-\right.$ $\left.x_{2} \ln C_{2}^{\text {Sat }}\right)$ against $x_{1} x_{2}, x_{1} x_{2}\left(x_{1}-x_{2}\right)$, and $x_{1} x_{2}\left(x_{1}-x_{2}\right)^{2}$ using experimental solubility data in the binary solvent mixture. The trained version of the model is capable of predicting unmeasured solubilities in the same solvent systems. The model provided accurate results for solubility data of anthracene in various nonaqueous binary solvents, and there is no need to evaluate its fitness abilities. ${ }^{3-7,10}$ Equation 1 could be extended to calculate the solute solubility in ternary solvent mixtures as ${ }^{11}$

$$
\begin{gathered}
\ln C_{\mathrm{m}}^{\mathrm{Sat}}=x_{1} \ln C_{1}^{\mathrm{Sat}}+x_{2} \ln C_{2}^{\mathrm{Sat}}+x_{3} \ln C_{3}^{\mathrm{Sat}}+ \\
x_{1} x_{2} \sum_{i=0}^{2} J_{i}\left(x_{1}-x_{2}\right)^{i}+x_{1} x_{3} \sum_{i=0}^{2} J_{i}^{\prime}\left(x_{1}-x_{3}\right)^{i}+ \\
x_{2} x_{3} \sum_{i=0}^{2} J_{i}^{\prime \prime}\left(x_{2}-x_{3}\right)^{i}+x_{1} x_{2} x_{3} \sum_{i=0}^{2} J_{i}^{\prime \prime}\left(x_{1}-x_{2}-x_{3}\right)^{i}
\end{gathered}
$$

where $x_{3}$ is the mole fraction of solvent $3, C_{3}^{\text {Sat }}$ denotes the mole per liter solubility of the solute in neat solvent 3 , and $J$ terms are model constants. Equation 2 is simplified to eq 1 when $x_{3}$ $=0$.

The aim of this work is to report the experimental solubility data of anthracene in a number of nonaqueous binary and ternary solvent mixtures at $298.2 \mathrm{~K}$. The mixtures include cyclohexanone + ethyl acetate, cyclohexanone + methanol, ethyl acetate + methanol, and cyclohexanone + ethyl acetate + methanol. Another attempt in the present work is to provide the Jouyban-Acree model constants for representing the solubility of anthracene in mixed solvents of cyclohexanone + ethyl acetate + methanol. 


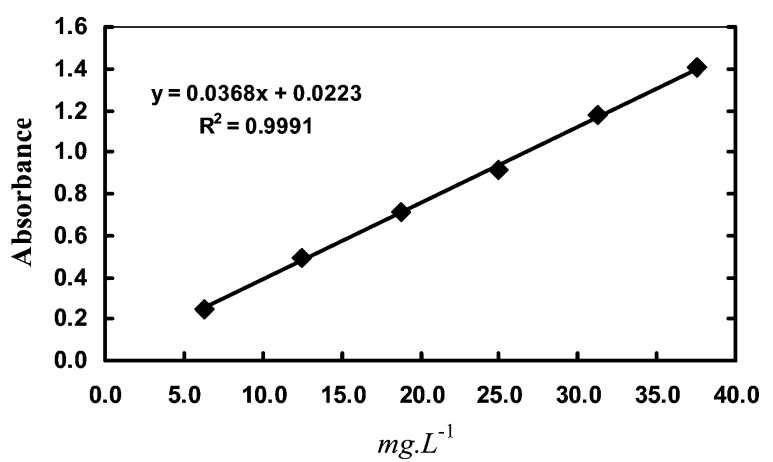

Figure 1. UV calibration curve for anthracene in 2-propanone at $356 \mathrm{~nm}$.

\section{Experimental Method}

Materials. Anthracene (0.960 in mass fraction) was purchased from Fluka and was recrystallized several times from ethyl acetate and then from 2-propanone (Merck, Germany) to yield a purified sample having a melting point of $488 \pm 0.2 \mathrm{~K}$. Its purity was also checked by thin layer chromatography. ${ }^{12}$ In addition, the measured solubilities in a number of monosolvents were compared with corresponding data from the literature. Cyclohexanone (0.990 in mass fraction), ethyl acetate (0.995 in mass fraction), and methanol (0.995 in mass fraction) were purchased from Merck (Germany).

Apparatus and Procedures. The binary and ternary solvent mixtures were prepared by mixing the appropriate volumes of

Table 1. Experimental and Calculated Molar Solubilities of Anthracene in Binary Solvent Mixtures at 298.2 K along with the Density of the Saturated Solutions

\begin{tabular}{|c|c|c|c|}
\hline$x_{1}$ & experimental & $\rho$ & calculated \\
\hline mole fraction & $\mathrm{mol} \cdot \mathrm{L}^{-1}$ & $\mathrm{~g} \cdot \mathrm{cm}^{-3}$ & $\mathrm{~mol} \cdot \mathrm{L}^{-1}$ \\
\hline \multicolumn{4}{|c|}{ Cyclohexanone (1) + Ethyl Acetate (2) } \\
\hline 0.000 & 0.049 & 0.901 & 0.049 \\
\hline 0.096 & 0.074 & 0.903 & 0.067 \\
\hline 0.193 & 0.078 & 0.909 & 0.081 \\
\hline 0.290 & 0.085 & 0.917 & 0.089 \\
\hline 0.389 & 0.094 & 0.921 & 0.095 \\
\hline 0.489 & 0.103 & 0.927 & 0.100 \\
\hline 0.589 & 0.107 & 0.931 & 0.106 \\
\hline 0.690 & 0.114 & 0.937 & 0.113 \\
\hline 0.793 & 0.124 & 0.942 & 0.122 \\
\hline 0.896 & 0.125 & 0.948 & 0.131 \\
\hline 1.000 & 0.136 & 0.953 & 0.136 \\
\hline \multicolumn{4}{|c|}{ Cyclohexanone (1) + Methanol (2) } \\
\hline 0.000 & 0.006 & 0.811 & 0.006 \\
\hline 0.042 & 0.013 & 0.831 & 0.010 \\
\hline 0.089 & 0.016 & 0.852 & 0.016 \\
\hline 0.144 & 0.022 & 0.876 & 0.024 \\
\hline 0.207 & 0.034 & 0.892 & 0.033 \\
\hline 0.281 & 0.039 & 0.906 & 0.044 \\
\hline 0.370 & 0.056 & 0.915 & 0.055 \\
\hline 0.477 & 0.068 & 0.928 & 0.067 \\
\hline 0.610 & 0.095 & 0.938 & 0.086 \\
\hline 0.779 & 0.111 & 0.949 & 0.120 \\
\hline 1.000 & 0.136 & 0.966 & 0.136 \\
\hline \multicolumn{4}{|c|}{ Ethyl Acetate (1) + Methanol (2) } \\
\hline 0.000 & 0.006 & 0.821 & 0.006 \\
\hline 0.044 & 0.009 & 0.831 & 0.008 \\
\hline 0.093 & 0.012 & 0.844 & 0.012 \\
\hline 0.149 & 0.016 & 0.856 & 0.016 \\
\hline 0.215 & 0.019 & 0.864 & 0.020 \\
\hline 0.291 & 0.026 & 0.872 & 0.025 \\
\hline 0.381 & 0.028 & 0.882 & 0.030 \\
\hline 0.489 & 0.038 & 0.891 & 0.035 \\
\hline 0.621 & 0.040 & 0.905 & 0.040 \\
\hline 0.787 & 0.045 & 0.918 & 0.046 \\
\hline 1.000 & 0.049 & 0.933 & 0.049 \\
\hline
\end{tabular}

Table 2. Experimental and Calculated Molar Solubilities of Anthracene in Cyclohexanone (1) + Ethyl Acetate (2) + Methanol (3) Solvent Mixtures at 298.2 K and the Density of the Saturated Solutions

\begin{tabular}{|c|c|c|c|c|}
\hline$x_{1}$ & $x_{2}$ & experimental & $\rho$ & calculated \\
\hline mole fraction & mole fraction & $\mathrm{mol} \cdot \mathrm{L}^{-1}$ & $\mathrm{~g} \cdot \mathrm{cm}^{-3}$ & $\mathrm{~mol} \cdot \mathrm{L}^{-1}$ \\
\hline 0.044 & 0.047 & 0.011 & 0.826 & 0.013 \\
\hline 0.056 & 0.233 & 0.027 & 0.853 & 0.027 \\
\hline 0.061 & 0.318 & 0.032 & 0.875 & 0.031 \\
\hline 0.084 & 0.702 & 0.042 & 0.855 & 0.047 \\
\hline 0.122 & 0.255 & 0.031 & 0.873 & 0.034 \\
\hline 0.176 & 0.222 & 0.041 & 0.875 & 0.040 \\
\hline 0.202 & 0.282 & 0.052 & 0.885 & 0.044 \\
\hline 0.218 & 0.227 & 0.050 & 0.888 & 0.046 \\
\hline 0.224 & 0.059 & 0.035 & 0.888 & 0.040 \\
\hline 0.246 & 0.128 & 0.042 & 0.883 & 0.047 \\
\hline 0.270 & 0.212 & 0.057 & 0.891 & 0.052 \\
\hline 0.301 & 0.315 & 0.055 & 0.900 & 0.059 \\
\hline 0.308 & 0.064 & 0.052 & 0.891 & 0.052 \\
\hline 0.339 & 0.444 & 0.069 & 0.862 & 0.071 \\
\hline 0.425 & 0.357 & 0.078 & 0.917 & 0.080 \\
\hline 0.690 & 0.090 & 0.105 & 0.932 & 0.102 \\
\hline
\end{tabular}

Table 3. Numerical Values of the Model Constants, Number of Data Points in Each Set $(N)$, and the Mean Deviation (MD) for the Calculated Solubilities of Anthracene in Cyclohexanone, Ethyl Acetate, and Methanol Mixtures and Their Overall Values

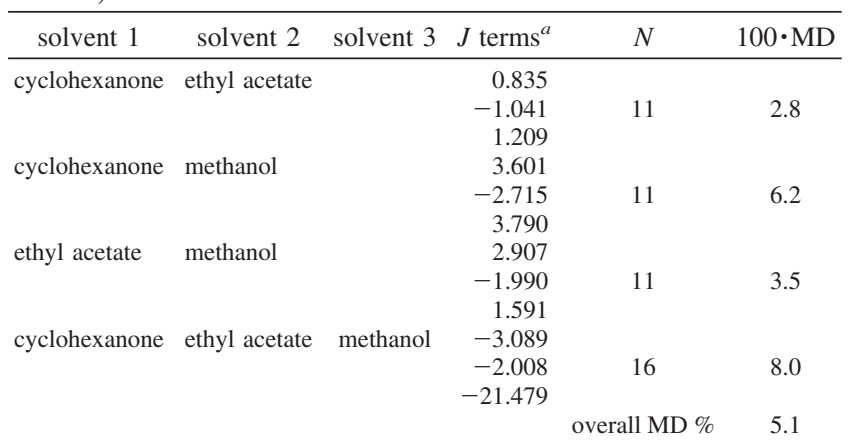

${ }^{a}$ The orders of $J$ terms are $J_{0}, J_{1}$, and $J_{2}$.

the solvents, and then the mole fractions of the solvents were computed considering the densities of the neat solvents. The solvent composition is calculated with the uncertainty of 0.005 mole fraction. The solubility of anthracene was determined by equilibrating an excess amount of the solid with the binary and ternary solvent mixtures at $298.2 \mathrm{~K}$ using a shaker (Behdad, Tehran, Iran) placed in an incubator equipped with a temperature-controlling system with the uncertainty of $0.2 \mathrm{~K}$ (Nabziran, Tabriz, Iran). Attainment of the equilibrium was verified by solubility measurements at different time intervals and reached after $72 \mathrm{~h}$. The solutions were filtered using hydrophobic Durapore filters $(0.45 \mu \mathrm{m}$, Millipore, Ireland) and then diluted with 2-propanone, and the absorbance of these solutions were recorded at $356 \mathrm{~nm}$ using a UV-vis spectrophotometer (Beckman DU-650, Fullerton, USA). The preliminary investigation showed that filter did not absorb the solute through the filtration process. Concentrations of the dilute solutions were determined from a UV absorbance calibration graph (see Figure 1) with the molar extinction coefficients of anthracene ranging from 667 $\varepsilon /\left(\mathrm{L} \cdot \mathrm{mol}^{-1} \cdot \mathrm{cm}^{-1}\right)$ to $713 \varepsilon /\left(\mathrm{L} \cdot \mathrm{mol}^{-1} \cdot \mathrm{cm}^{-1}\right)$ for the anthracene concentrations ranging from $(0.00035$ to 0.0021$) \mathrm{mol} \cdot \mathrm{L}^{-1}$. Each experimental data point is an average of at least three experimental measurements with the measured $\mathrm{mol} \cdot \mathrm{L}^{-1}$ solubilities being reproducible to within the mean relative standard deviations (RSDs) of $1.1 \%$ (the RSDs vary between (0.2 and 5.0) $\%$ ). Calculated standard deviations ranged from $\sigma_{n-1}=0.00005$ to $\sigma_{n-1}=0.00193 \mathrm{~mol} \cdot \mathrm{L}^{-1}$. Densities of the saturated solutions 
were measured using a $5 \mathrm{~mL}$ pycnometer with the uncertainty of $0.001 \mathrm{~g} \cdot \mathrm{cm}^{-3}$.

Computational Methods. The $J_{i}$ terms of the Jouyban-Acree model were computed using a no-intercept least-squares analysis. The model constants of eqs 1 and 2 along with the experimental solubility of anthracene in monosolvents were employed to calculate the solubilities. All calculated $C_{\mathrm{m}}^{\mathrm{Sat}}$ solubilities were compared with the corresponding experimental values, and the mean deviation (MD) was calculated as an accuracy criterion by:

$$
\mathrm{MD}=\frac{1}{N} \sum \frac{\mathrm{l}\left(C_{\mathrm{m}}^{\mathrm{Sat}}\right)_{\text {predicted }}-\left(C_{\mathrm{m}}^{\mathrm{Sat}}\right)_{\text {experimental }} \mathrm{I}}{\left(C_{\mathrm{m}}^{\mathrm{Sat}}\right)_{\text {experimental }}}
$$

in which $N$ is the number of data points in each set.

\section{Results and Discussion}

Tables 1 and 2 list the experimental solubilities of anthracene, along with the back-calculated solubilities using eqs 1 and 2 and the densities of the respective saturated solutions. There were good agreements between the published ${ }^{13}$ solubilities of anthracene at $298.2 \mathrm{~K}$ in ethyl acetate $\left(0.049 \mathrm{~mol} \cdot \mathrm{L}^{-1}\right)$ and methanol $\left(0.006 \mathrm{~mol} \cdot \mathrm{L}^{-1}\right)$ and the experimental anthracene solubilities in ethyl acetate $\left(0.049 \mathrm{~mol} \cdot \mathrm{L}^{-1}\right)$ and methanol $(0.006$ $\left.\mathrm{mol} \cdot \mathrm{L}^{-1}\right)$ reported in this study. The solubility of anthracene in binary and ternary solvent mixtures was calculated using eqs 1 or 2 . The calculated model constants and MD values were computed and listed in Table 3 in which the solubilities were calculated with a reasonable MD (5.1\%), and this finding is in agreement with previous results. ${ }^{3-5}$

\section{Literature Cited}

(1) Jacob, J. The Significance of Polycyclic Aromatic Hydrocarbons as Environmental Carcinogens. 35 Years Research on PAH- A Retrospective. Polycyclic Aromat. Compd. 2008, 28, 242-272.

(2) Jouyban, A. Review of the Cosolvency Models for Predicting Solubility of Drugs in Water-Cosolvent Mixtures. J. Pharm. Pharm. Sci. 2008, $11,32-58$.
(3) Zvaigzne, A. I.; Teng, I. L.; Martinez, E.; Trejo, J.; Acree, W. E., Jr. Solubility of Anthracene in Binary Alkane + 1-Propanol and Alkane + 1-Butanol Solvent Mixtures. J. Chem. Eng. Data 1993, 38, 389392.

(4) Zvaigzne, A. I.; McHale, M. E. R.; Powell, J. R.; Kauppila, A. S. M.; Acree, W. E., Jr. Solubility of Anthracene and Pyrene in Binary Alcohol + Alcohol Solvent Mixtures. J. Chem. Eng. Data 1995, 40, $1273-1275$.

(5) Toro, A.; Acree, W. E., Jr. Solubility of Anthracene in Binary Alcohol + Ethyl Acetate Solvent Mixtures. Phys. Chem. Liq. 2002, 40, 327335

(6) Corella, M.; Wolcott, K.; Carrillo, M.; Acree, W. E., Jr. Solubility of Anthracene in Binary Diisopropyl Ether + Alcohol Solvent Mixtures at 298.15 K. J. Chem. Eng. Data 2007, 52, 929-931.

(7) Proctor, A.; Blake-Taylor, B. H.; Acree, W. E., Jr. Solubility of Anthracene in Ternary Cyclohexane + Propanol + 2-Methyl-1propanol and Cyclohexane + Butanol + 2-Methyl-1-propanol Mixtures. J. Chem. Eng. Data 2008, 53, 2910-2912.

(8) Shayanfar, A.; Soltani, S.; Jabbaribar, F.; Tamizi, E.; Acree, W. E., Jr.; Jouyban, A. Solubility of Anthracene in Ternary Solvent Mixtures of 2,2,4-Trimethylpentane + 2-Propanone + Alcohols at $298.15 \mathrm{~K}$. J. Chem. Eng. Data 2008, 53, 890-893.

(9) Shayanfar, A.; Soltanpour, S.; Jabbaribar, F.; Hamidi, A. A.; Acree, W. E., Jr.; Jouyban, A. Solubility of Anthracene in Quaternary Solvent Mixtures of 2,2,4-Trimethylpentane + 2-Propanone + Methanol + Alcohols at 298.15 K. J. Chem. Eng. Data 2008, 53, 2250-2253.

(10) Jouyban, A.; Khoubnasabjafari, M.; Chan, H. K.; Clark, B. J.; Acree, W. E., Jr. Solubility Prediction of Anthracene in Mixed Solvents Using a Minimum Number of Experimental Data. Chem. Pharm. Bull. 2002, $50,21-25$.

(11) Jouyban, A.; Khoubnasabjafari, M.; Hamidi, A. A.; Acree, W. E., Jr. $\mathrm{Ab}$ initio Solubility Prediction of Non-electrolytes in Ternary Solvents Using a Combination of Jouyban-Acree and Abraham Models. Asian J. Chem. 2008, 53, 574-577.

(12) Loncar, E. S.; Kolarov, L. A.; Malbasa, R. V.; Skrbic, B. D. Qualitative TLC Determination of Some Polycyclic Aromatic Hydrocarbons in Sugar-Beet. J. Serb. Chem. Soc. 2005, 70, 1237-1242.

(13) Acree, W. E., Jr.; Abraham, M. H. Solubility Predictions for Crystalline Nonelectrolyte Solutes Dissolved in Organic Solvents Based Upon the Abraham General Solvation Model. Can. J. Chem. 2001, 79, 14661476.

Received for review October 19, 2009. Accepted April 28, 2010.

JE900852T 